\title{
Outpatient psychotherapy for home-living vulnerable older adults with depression: study protocol of the PSY-CARE trial
}

\author{
Paul Gellert ${ }^{1 *}$ D, Ann-Kristin Beyer ${ }^{1}$, Christina Tegeler ${ }^{2}$, Claudia Vathke ${ }^{2}$, Johanna Nordheim¹,
} Adelheid Kuhlmey ${ }^{1}$ and Eva-Marie Kessler ${ }^{2}$

\begin{abstract}
Background: There is a need to improve psychotherapeutic approaches to treatment for vulnerable older adults with depression in terms of both clinical practice and health care supply. Against this background, PSY-CARE is testing the feasibility and effectiveness of outpatient psychotherapy for home-living older adults in need of care with depression in Berlin, Germany, and neighboring suburban areas.

Methods: In a two-arm single-center pragmatic randomized controlled trial (RCT), manual-guided outpatient psychotherapy will be compared to brief psychosocial counseling. The study population will be compromised of older adults with clinically significant depressive symptoms who have a long-term care grade, as assessed by the German compulsory state nursing care insurance. In the intervention group, individual cognitive-behavioral psychotherapy tailored to the specific needs of this population will be offered by residential psychotherapists as part of the regular healthcare service. In the active control group, participants will receive individual psychosocial telephone counselling and a self-help guide. The planned sample size is $N=130$ ( $n=65$ participants per group). The reduction of depressive symptoms (primary outcome) as well as the maintaining of activities of daily living, quality of life, and functioning will be assessed with questionnaires provided at baseline, after the end of the intervention and after three months. Feasibility and process evaluation will be conducted qualitatively based on documentation and interviews with psychotherapists, gatekeepers and the participants.
\end{abstract}

Discussion: PSY-CARE investigates the potentials and limitations of providing outpatient psychotherapeutic treatment meeting the demands of vulnerable home-living older adults with depression under the real conditions of the health care system. The study will provide practical implications to improve access to and quality of outpatient psychotherapy for this poorly supplied population.

Trial registration: The trial is registered at ISRCTN55646265; February 15, 2019.

Keywords: Depression in older adults, Homecare, Psychotherapy, Randomized controlled trial, Pragmatic clinical trial

\footnotetext{
* Correspondence: paul.gellert@charite.de

${ }^{1}$ Institute for Medical Sociology and Rehabilitation Science, Charité -

Universitätsmedizin Berlin, Charitéplatz 1, 10117 Berlin, Germany

Full list of author information is available at the end of the article
}

(c) The Author(s). 2021, corrected publication 2021. Open Access This article is licensed under a Creative Commons Attribution 4.0 International License, which permits use, sharing, adaptation, distribution and reproduction in any medium or format, as long as you give appropriate credit to the original author(s) and the source, provide a link to the Creative Commons licence, and indicate if changes were made. The images or other third party material in this article are included in the article's Creative Commons licence, unless indicated otherwise in a credit line to the material. If material is not included in the article's Creative Commons licence and your intended use is not permitted by statutory regulation or exceeds the permitted use, you will need to obtain permission directly from the copyright holder. To view a copy of this licence, visit http://creativecommons.org/ licenses/by/4.0/. The Creative Commons Public Domain Dedication waiver (http://creativecommons.org/publicdomain/zero/1. 0/) applies to the data made available in this article, unless otherwise stated in a credit line to the data. 


\section{Background}

Depression is a common phenomenon among vulnerable older adults. Compared with the general older population, community-based studies have consistently documented at least two or three times higher rates of depression among older adults with chronic physical conditions, cognitive deficits and/ or sensory limitations, i.e. conditions that often along with functional impairment and dependence on informal and formal support systems for medical care, transport and other essential needs (e.g., [1-5]). Conversely, depression has devastating consequences for older individuals including an increased risk of morbidity and suicide, decreased physical, cognitive and social functioning, all of which are in turn associated with higher rates of service utilization, premature institutionalization and mortality $[6,7]$.

Meta-analyses and systematic reviews have provided extensive evidence that psychotherapeutic interventions are effective for treating depression in the older population, with strongest evidence for cognitive and behavioral therapy (CBT), life review therapy (LR) and problem-solving therapy (PST) [8, 9]. However, vulnerable older adults with depression including very old, frail and care-dependent older adults were mostly excluded from these studies [10], despite advances for psychotherapeutic research in the context of specific comorbid conditions including chronic obstructive pulmonary disease (COPD), heart failure, Parkinson's disease, stroke, early stages of dementia and suicidal ideations [for an overview, see [11]]. As a result, psychotherapy for this rapidly growing population is understudied in terms of clinical practice, feasibility, efficiency and effectiveness.

The described deficiencies in research are also mirrored in the massive shortage of mental health care supply for vulnerable older adults with depression [e.g., [12]]. Under the current conditions of health care systems, referrals to outpatient psychotherapy very seldom succeed due to health care providers' pessimistic attitudes towards vulnerable older patients' treatability, patients' inaccessibility, shortage of psychotherapists qualified to work with older adults, and costs (e.g., [1315]). For example, although outpatient psychotherapy is integral part of public and private health care in Germany, ambulatory psychotherapy is de facto nonexisting or only in very exceptional cases among vulnerable older people in need of care [16]. The fact that vulnerable older adults' mental health care needs are obviously largely unmet is even more problematic as most of them have positive attitudes towards psychotherapy or even prefer psychotherapy to pharmacotherapy, contrary to what is often believed $[17,18]$.

The significant correlation between depression and impaired quality of life together with increasing numbers of vulnerable older adults and the very poor supply reality highlight the importance of developing and rigorously evaluating appropriate psychotherapeutic approaches, including outpatient psychotherapy for homeliving vulnerable older adults.

When it comes to clinical practice, existing studies on mental health services for homebound older adults and nursing-home participants has clearly shown the necessity of gatekeepers (such as aging services networks for patients to gain access to mental health care providers), interprofessional collaboration, caregiver involvement and in-home treatment (for patients who cannot freely leave their home $[19,20])$. Furthermore, vulnerable older patients have been shown to profit from CBT and PST through encouragement of adaptive coping skills together with behavioral activation and increased exposure to pleasant events (e.g., [21-23]). Finally, research on LR in long-term care has shown the importance of reminiscence and biographical interventions for patients in order to identify coping skills in their biography as well as to find hope and purpose in life (e.g., $[23,24])$.

\section{Objectives}

PSY-CARE investigates the feasibility and effectiveness of individual outpatient psychotherapy within the regular German health care system for home-living older adults in need of care with clinically significant depression. Outpatient psychotherapy tailored to the specific needs of this population is provided based on a treatment manual by certified resident CBT psychotherapists with extensive professional experience in geriatric or geropsychiatric settings.

In terms of the effectiveness of the intervention, we hypothesized that psychotherapy leads to a greater reduction of depressive symptoms, which we considered the primary outcome, compared to an alternative intervention, which encompassed brief psychosocial counselling (Hypothesis 1). Further, we hypothesized that psychotherapy will be associated with a relative difference at follow-up in the secondary outcomes - i.e., quality of life, activities of daily living, functioning and subjective health - in the intervention condition compared with the control condition (Hypothesis 2). More specifically, we assume maintenance of activities of daily living and quality of life, and functioning/ subjective health in the intervention condition relative to the control condition, which we assume shows a decline. Further, in this trial treatment response $(50 \%$ or greater reduction in depressive symptoms from baseline to follow up) and remission [defined as number of individuals who scoring below the cut-off of 10 points at the Geriatric Depression Scale (GDS; [25, 26] at follow up] will be used as secondary outcomes.

In terms of the feasibility of the intervention under the real conditions of the German health care system, a wide 
range of approaches to recruit participants of this hardto-reach population is used and effective versus noneffective recruitment methods are documented. In addition, we aim to explore possibilities and constraints of psychotherapists' clinical practice of working with home-living vulnerable older adults including their professional experiences with home treatment in case of participants' limited mobility, as well as collaboration with other healthcare professionals, especially with general practitioners and formal or informal caregivers.

\section{Methods/design}

\section{Trial design}

PSY-CARE is a single-center interventional study, using a pragmatic randomized controlled design with nested qualitative research, comparing individual psychotherapy with an active control condition. The intervention condition will include manual-guided psychotherapy provided by gerontologically trained resident psychotherapists [27]. The active control condition will include individual telephone counselling carried out by project staff members as well as a self-help guide for participants and their caregivers [28]. The flow chart of the study design is depicted in Fig. 1. The schedule of enrolment, interventions, and assessments is shown in Table 1.

PSY-CARE is going to be located in Berlin, Germany, and neighboring suburban areas. The study center will be located at the Department of Psychology, MSB Medical School Berlin. The method center (i.e., external evaluation) will be located at the Institute for Medical Sociology and Rehabilitation Science, Charité - Universitätsmedizin Berlin.

\section{Participants, interventions, and outcomes Study setting}

The psychotherapy intervention will take place in psychotherapy practices or - in case of a participant's impaired mobility or confinement to bed - at participants' homes (i.e., in that case psychotherapists will be traveling to participants' homes). The control group will receive treatment via telephone (psychosocial counseling) and postal service (self-help literature; audiobook or printed version).

\section{Eligibility criteria}

Participants will be included in the study if they [1] are 60 years and older (women/ men) [2]; are living at home (Berlin, Germany) [3]; have a long-term care grade, as assessed by the German compulsory state nursing care insurance ( $1=$ little impairment to $5=$ hardship case; see [29]) [4]; show clinically relevant depressive symptoms (major depression; adjustment disorder with depressive symptoms; dysthymia); and [5] are willing to participate in one of the two treatments. Exclusion criteria will be moderate to severe dementia as well as delirium, acute psychosis or other cognitive disorders, mania or hypomania or terminal stage of a disease. Further reasons for exclusion will be currently receiving psychotherapy, communication difficulties and visual or hearing impairments that would severely affect an individual's capability to take part in the study. Reasons for participant exclusion will be recorded.

\section{Interventions}

Participants included in the study will be randomly assigned to one of two treatment groups (intervention condition versus active control condition). the intervention condition, outpatient short-term psychotherapy tailored to the specific needs of vulnerable older adults, will be offered by residential CBT psychotherapists with extensive geriatric and geropsychiatric experience as part of the regular healthcare service. After the taking of an anamnesis, a medical examination by a general practitioner or a medical specialist and test sessions, participants will be treated in up to 24 therapy sessions. Psychotherapeutic treatment, as described in the PSYCARE manual, follows the regular CBT rationale, but is augmented by psychotherapeutic techniques of a) reminiscence, b) empowerment and c) inclusion of participants' social systems, based on existing effective psychotherapeutic approaches for vulnerable older adults with depression (see above). Before participating in PSYCARE, psychotherapists will be trained in the assessment of depression in vulnerable older adults and the application of the PSY-CARE Manual.

The active control condition will include individual telephone counselling carried out by project staff members as well as self-help literature for participants and their caregivers. The telephone counselling will be offered two times per participant and per caregiver by members of the project team (all trained certified psychotherapists). In addition, control group participants and their caregivers will receive a print or audio version of a self-help guide based on existing literature on depression in later life [e.g., [30]], which will include psychoeducation and self-help instructions. For participants in the control condition, regular psychotherapy will not be retained if desired.

Critical events for intervention or control participants will be recorded. In order to improve and monitor the adherence to intervention protocol, psychotherapists will be regularly supervised by PSY-CARE supervisors. The project team will stay in contact with participants via telephone or letters on a regular basis.

\section{Outcomes}

The primary outcome variable is the reduction of depressive symptoms of the participants assessed by the 


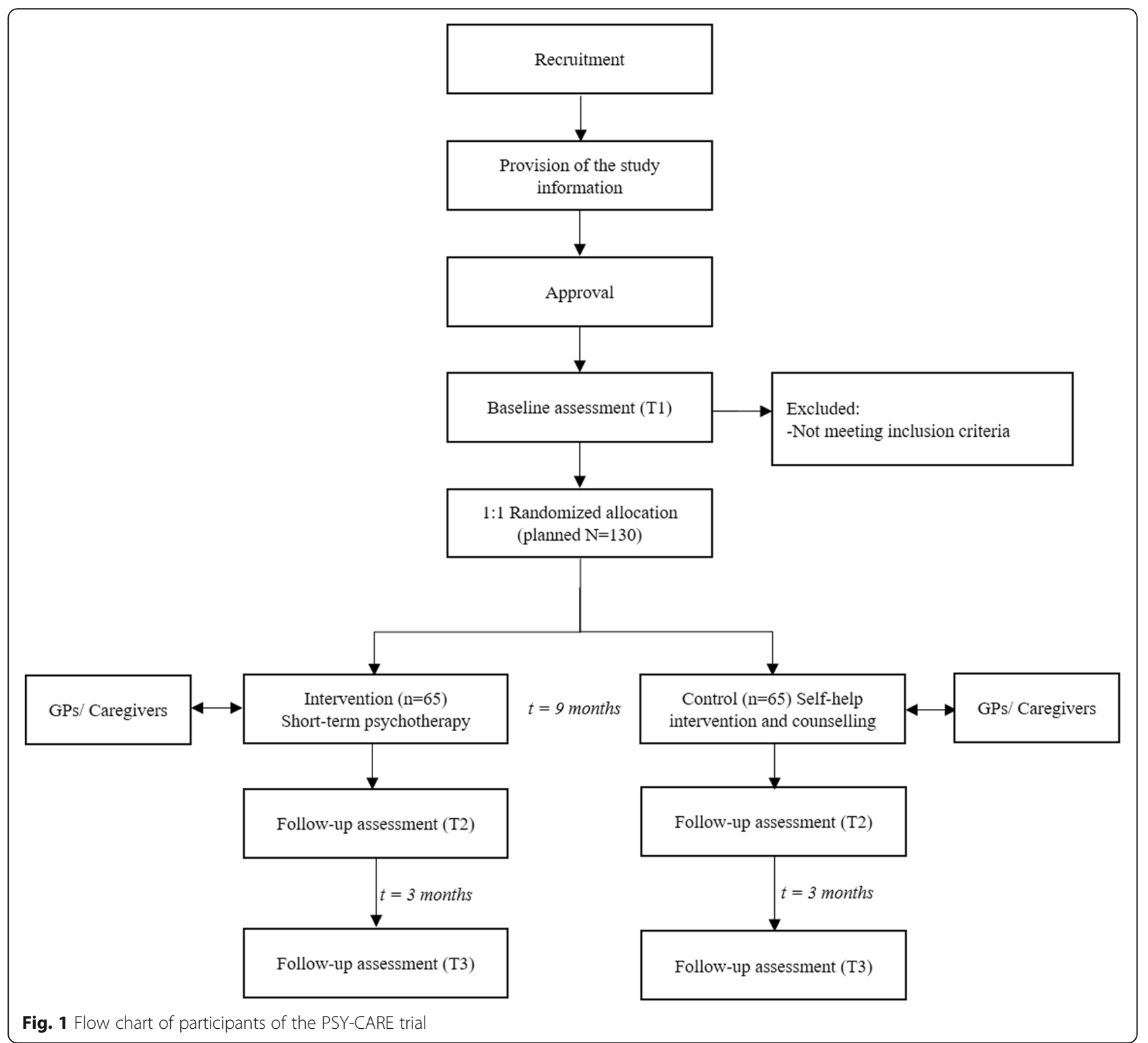

12-item Geriatric Depression Scale (GDS [31, 32];) at baseline (T1), directly after the intervention (T2), and at follow-up after 3 months (T3) compared to baseline. Total scores range from 0 to 12 , a score of 4 or more indicates the presence of clinically relevant depressive symptoms.

Secondary outcome variables are participants' [1] quality of life measured by a single item from the World Health Organization Quality of Life in old age, i.e. WHOQOL-OLD [2, 33]; the diagnose of depressive symptoms (major depression; adjustment disorder with depressive symptoms; dysthymia; F32, F33, F34, F43), assessed by a German structured clinical interview for psychological diseases (Mini-DIPS [34];) as well as depressive symptoms (and comorbid anxiety) assessed with the Hospital Anxiety and Depression Scale (HADS [35];) [3] Activities of daily living and functioning measured using the Barthel-Index and the IADL-Scale [36, 37]; and [4] self-rated health measured by a single item asking "How would you estimate your health status in the last 4 weeks?" on a 6 -point Likert scale from $1=$ "very poor" to $6=$ "excellent". Treatment response $(50 \%$ or greater reduction in depressive symptoms from baseline to follow up) and remission (defined as number of individuals who scoring below the cut-off of 10 points at the GDS at follow up) will be used as secondary outcomes. All secondary outcomes will be assessed at Baseline (T1), after the intervention (T2), as well as at follow-up after 3 months (T3).

\section{Sample size}

For sample size estimation, a difference in depression (i.e., primary outcome) between intervention and control 
Table 1 Schedule of enrolment, interventions, and assessments of the PSY-CARE trial

\begin{tabular}{|c|c|c|c|c|c|c|c|c|}
\hline \multirow[b]{3}{*}{ TIMEPOINT } & \multicolumn{8}{|c|}{ STUDY PERIOD } \\
\hline & \multirow[t]{2}{*}{ Enrolment } & \multirow{2}{*}{$\begin{array}{c}\text { Baseline } \\
T 1\end{array}$} & \multirow[t]{2}{*}{ Allocation } & \multicolumn{5}{|c|}{ Post-allocation } \\
\hline & & & & $m_{1}$ & $m \ldots$ & $m_{24}$ & $T 2$ & $T 3$ \\
\hline \multirow{4}{*}{$\begin{array}{r}\text { ENROLMENT: } \\
\text { Eligibility screen } \\
\text { Informed consent } \\
\text { Allocation } \\
\end{array}$} & & & & & & & & \\
\hline & $\mathrm{X}$ & & & & & & & \\
\hline & $\mathrm{X}$ & & & & & & & \\
\hline & & & $X$ & & & & & \\
\hline \multirow{3}{*}{$\begin{array}{r}\text { INTERVENTIONS: } \\
\text { Individual psychotherapy }^{\mathrm{a}} \\
\text { Active control condition }^{\mathrm{b}}\end{array}$} & & & & & & & & \\
\hline & & & & $\leftarrow$ & & $\rightarrow$ & & \\
\hline & & & & $\mathrm{X}$ & & $\mathrm{X}$ & & \\
\hline \multicolumn{9}{|l|}{ ASSESSMENTS: } \\
\hline \multicolumn{9}{|l|}{ Primary outcome } \\
\hline GDS & & $\mathrm{X}$ & & & & & $\mathrm{X}$ & $\mathrm{X}$ \\
\hline \multicolumn{9}{|l|}{ Secondary outcomes } \\
\hline WHOQOL-OLD & & $\mathrm{X}$ & & & & & $\mathrm{X}$ & $\mathrm{X}$ \\
\hline Mini-DIPS & & $\mathrm{X}$ & & & & & $\mathrm{X}$ & $\mathrm{X}$ \\
\hline HADS & & $\mathrm{X}$ & & & & & $\mathrm{X}$ & $\mathrm{X}$ \\
\hline BI & & $\mathrm{X}$ & & & & & $\mathrm{X}$ & $\mathrm{X}$ \\
\hline IADL & & $\mathrm{X}$ & & & & & $\mathrm{X}$ & $\mathrm{X}$ \\
\hline Self-rated health & & $X$ & & & & & $\mathrm{X}$ & $\mathrm{X}$ \\
\hline Post session assessment & & & & $\mathrm{X}$ & $\mathrm{X}$ & $\mathrm{X}$ & & \\
\hline
\end{tabular}

a Three probationary sessions and up to 24 therapy sessions

${ }^{\mathrm{b}}$ Individual telephone counselling, which is offered two times per participant and per caregiver carried out by trained staff members as well as self-help literature for participants and their caregivers

GDS Geriatric Depression Scale, WHOQOL-OLD single item of the World Health Organization Quality of Life in OLD age. Mini-DIPS German structured clinical interview for the diagnose of depressive symptoms. HADS Hospital Anxiety and Depression Scale. BI Barthel-Index (Activities of daily living). IADL Instrumental Activities of Daily Living-Scale at. $T 1$ Time point 1, baseline. T2 Time point 2, after the intervention

group at follow-up of medium effect size is considered as clinically relevant (Cohen's $d=0.5$ ), which also corresponds to finding from a recent meta-analysis [8]. The sample estimation (test family: t-test for independent samples) with Gpower 3.1 [38] yielded an estimated minimum sample size of the two groups of $n=51$ at a Type I error rate of alpha $=0.05$ and a statistical power of 1-beta $=0.80$ individuals. If a dropout rate of $20 \%$ is taken into account [39], as was the case in comparable studies, the final rounded sample size per group is $n=$ 65 , or $N=130$ persons in total. While $20 \%$ of drop out is common in psychotherapy trails for older adults, considering the vulnerability of our sample of vulnerable participants, we will be collecting further participants to guarantee sufficient statistical power at follow up.

\section{Recruitment of psychotherapists}

The recruitment of resident psychotherapists who volunteer to participate in PSY-CARE is supported by the biggest Association for Behavior Therapy in Germany (German Society for Behavioral Therapy) and includes several offers in newsletters, online mailing lists and distribution of flyers. Certified CBT psychotherapists with extensive work experience in geriatric and/or geropsychiatric settings are included into the pool of PSY-CARE psychotherapists. In addition, they receive a two days training course, conducted by the principal investigator (PI) [40]. They will receive an expense allowance of $€ 1000$ for their participation in the study, in addition to their regular fees for psychotherapeutic services within the regular health care system.

\section{Recruitment of participants and the treatment team}

Recruitment takes place via self-referral as well as gatekeeper referral and uses a wide range of approaches including distributions of flyers and posters, public talks, presentations within institutions, advertisements in online newsletters of collaborating institutions in the health and care sector and media reports in newspapers and magazines. In addition, professionals working with vulnerable home-living older adults on a day-to-day basis in ambulatory care services, medical practices, geriatric and geropsychiatric clinics and counseling centers were recruited via phone call, e-mail or mail. Gatekeepers are given flyers and reminder letters to prompt referrals. In order to participate in the study, an informed consent 
sheet is signed and participants receive a copy of the signed documents. Neither participants nor gatekeepers will receive any direct financial compensation. The main caregiver of the participant (professional caregiver or family member) is included in the study, if the participants give their written consent for participation.

\section{Assignment of interventions \\ Allocation and blinding}

Participants will be randomized to the two treatment arms. A computer-assisted randomization to either intervention or control condition with 1:1 ratio is performed by the method center, using $\mathrm{R}$ v3.2.1 [41, 42] and the package "blockrand" (block randomization with block sizes 4, 6, and 8). Stratifications is not undertaken. In order to ensure allocation concealment, central randomization is used at the participant level, where the recruiting person got sequentially numbered, opaque, sealed envelopes from the method center. The creation of the random assignment sequence, the enrollment participants and the assignment of the participants to the interventions will be strictly separated. The project staff involved in evaluating the results will be blinded to the intervention allocation.

\section{Data collection, management and statistical analysis}

Standardized assessments will be carried out at baseline, directly after the intervention and at a follow-up after 3 months. The quantitative assessment (Hypotheses 1 and 2) among participants will be carried out by two trained study nurses (clinical psychologists with advanced clinical training). The questionnaires will be conducted as computer assessed interviews using a secure laptop and will take up to a maximum of two hours per participant. Data collection will be monitored by the method center and later automatically transferred to SPSS v25 (IBM, Cary, Ind.). When selecting the measuring instruments, attention was paid to the objectivity, reliability, and validity of the assessments.

Psychotherapists will also complete questionnaires in paper-pencil format and later transferred to a SPSS v25 data mask. Missing data will be handled using multiple imputation. Furthermore, qualitative data will be collected from psychotherapists, gatekeepers and older participants. Data will be stored and analyzed at the method center. Only the evaluation team will have access to the data, as defined in the data protection concept as part of the ethics approval.

The evaluation based on participant data will be carried out by means of multivariate longitudinal methods (random effects models). Both intention-to-treat analyzes and completers analyzes will be carried out. A post hoc effectiveness analysis of three potentially moderating characteristics of those in need of care will be considered: (a) degree of cognitive impairment, (b) drug use, and (c) late-onset versus early onset depression. In addition to effect sizes, remission and response rates (Reliable Change Index; RCI) will be determined.

Alongside the effectiveness trial, qualitative data gathered over recruitment as well as treatment processes will be analyzed in order to provide a systematic analysis of the therapeutic, structural and organizational possibilities and barriers regarding the realization of outpatient psychotherapy within the context of interprofessional cooperation under real-life conditions of the German health care system. Specifically, documentation of approaches to access vulnerable older adults will be compared to one another in order to identify successful versus non-successful approaches. In terms of practical implications, qualitative methods will be used in order to analyze interview and focus group data of psychotherapists' experiences of providing psychotherapy for their patients. In addition, qualitative methods are used to analyze how patients experience the psychotherapeutic process and its effectiveness. Quantitative and qualitative results will finally be combined (method triangulation).

\section{Trial status}

Recruitment/ enrolment began 25/02/2019 (i.e., first participant in). Currently, we are estimating that the assessment phase will continue until September 2021 (i.e., last participant out) when the last psychotherapy session and T3 assessment has been completed.

\section{Ethics and dissemination \\ Research ethics approval}

This research was approved by the Ethics Committee of the Medical School Hamburg (25/10/2018; MSB-2018/20).

\section{Protocol amendments}

Important protocol modifications will be reported in the trial paper.

\section{Consent or assent}

PSY-CARE participants and psychotherapists will receive comprehensive information material on the research project and the trial. Written informed consent to take part in the study will be obtained from participants and certified psychotherapists prior to data collection.

\section{Declaration of interests}

The authors declare that they have no competing interests. The funder had no role in the design of this study and will not have any role during its execution, analyses, interpretation of the data, or decision to submit results. 


\section{Access to data}

The project team will be given access to the cleaned data set. The datasets generated during and analyzed during the current study will be stored in a non-publically available repository. The access information is available from the PI and the corresponding author on reasonable request. To ensure confidentiality, data dispersed to project team members will be blinded of any identifying participant information.

\section{Dissemination policy}

Publications are planned in a high-impact peer-reviewed journal.

\section{Discussion}

PSY-CARE is a large international study using a pragmatic randomized controlled design for testing the feasibility and effectiveness of outpatient psychological therapy for home-living vulnerable older adults with clinically significant depression. Going beyond extant psychotherapy studies, a systematic analysis of the therapeutic, structural and organizational possibilities and barriers regarding the realization of outpatient psychotherapy under real-life conditions of the German healthcare system is provided which may in part be transferable to other countries. Overall, the present trial aims to provide real-world evidence on the provision of psychotherapeutic treatment for home-living vulnerable older adults, with the potential of providing practical implications to improve access to and quality of outpatient psychotherapy.

\section{Supplementary information}

Supplementary information accompanies this paper at https://doi.org/10. 1186/s12877-020-01661-1.

Additional file 1. PSY-CARE trial: Update to the study protocol.

\begin{abstract}
Abbreviations
CBT: Cognitive and behavioral therapy; COPD: Chronic obstructive pulmonary disease; DFG: German Research Foundation; G-BA: Gemeinsamer Bundesausschuss [Fund of the German Federal Joint Committee]; GDS: Geriatric Depression Scale; HADS: Hospital Anxiety and Depression Scale; ID: Identity number; ISRCTN: International Standard Randomised Controlled Trial Number; LR: Life review therapy; Mini-DIPS: Diagnostisches Kurzinterview bei psychischen Störungen [Diagnostic brief Interview for Psychological Diseases]; MSB: Medical School Berlin; PST: Problem-solving therapy; RCl: Reliable change index; RCT: Randomized controlled trial; PSYCARE: Psychological counselling and therapy for treating depression in homebound older adults; WHOQOL-OLD: World Health Organization Quality of Life in OLD age
\end{abstract}

\section{Acknowledgements}

We acknowledge support from the German Research Foundation (DFG) and the Open Access Publication Funds of Charité - Universitätsmedizin Berlin.

\section{Authors' contributions}

EMK (principal investigator) conceptualized the study. EMK, AKB, and PG were major contributors in writing the first draft of the manuscript. All authors (PG, AKB, CT, CV, JN, AK, EMK) made substantial suggestions to further qualify the first version of the manuscript. $P G$ conducted the power analysis and wrote the statistical methods section. All authors (PG, AKB, CT, $\mathrm{CV}, \mathrm{JN}, \mathrm{AK}, \mathrm{EMK})$ substantively revised the manuscript and all authors (PG, $A K B, C T, C V, J N, A K, E M K$ ) read and approved the final manuscript. Finally, all authors ( $P G, A K B, C T, C V, J N, A K, E M K)$ confirm that they are personally accountable for their own contributions and ensure that questions related to the accuracy or integrity of any part of the work, even ones in which the author was not personally involved, are appropriately investigated, and resolved.

\section{Funding}

The study is funded by the German Innovation Fund of the German Federal Joint Committee (Gemeinsamer Bundesausschuss, G-BA; 01VSF17048). Principal investigator is Eva-Marie Kessler. The funder had no role in the design of this study and will not have any role during its execution, analyses, interpretation of the data, or decision to submit results. Open access funding provided by Projekt DEAL.

\section{Availability of data and materials}

The datasets generated during and analyzed during the current study will be stored in a non-publically available repository. The access information is available from the $\mathrm{Pl}$ and the corresponding author on reasonable request. To ensure confidentiality, data dispersed to project team members will be blinded of any identifying participant information.

\section{Ethics approval and consent to participate}

This research was approved by the Ethics Committee of the Medical School Hamburg (25/10/2018; MSB-2018/20). Written informed consent was obtained from study participants. The trial was prospectively registered at the ISRCTN registry (International Standard Randomised Controlled Trial Number) with study identity number ISRCTN55646265.

\section{Consent for publication}

Not applicable - This protocol contains no individual person's data.

\section{Competing interests}

The authors declare that they have no conflict of interest.

\section{Author details}

${ }^{1}$ Institute for Medical Sociology and Rehabilitation Science, Charité Universitätsmedizin Berlin, Charitéplatz 1, 10117 Berlin, Germany. ${ }^{2}$ MSB Medical School Berlin, Department of Psychology, Rüdesheimer Str. 50, 14197 Berlin, Germany.

Received: 15 January 2020 Accepted: 22 July 2020

Published online: 05 August 2020

\section{References}

1. Bruce ML, McAvay GJ, Raue PJ, Brown EL, Meyers BS, Keohane DJ, et al. Major depression in elderly home health care patients. Am J Psychiatr. 2002; 159(8):1367-74

2. Choi NG, Teeters M, Perez L, Farar B, Thompson D. Severity and correlates of depressive symptoms among recipients of meals on wheels: age, gender, and racial/ethnic difference. Aging Ment Health. 2010;14(2):145-54.

3. Rowe JL, Conwell Y, Schulberg HC, Bruce ML. Social support and suicidal ideation in older adults using home healthcare services. Am J Geriatr Psychiatry. 2006;14(9):758-66..

4. Hyer L. Depression in long-term care. Clin Psychol Sci Pract. 2005;12(3): 280-99.

5. Xiang X, Leggett A, Himle JA, Kales HC. Major depression and subthreshold depression among older adults receiving home care. Am J Geriatr Psychiatry. 2018;26(9):939-49.

6. Fiske A, Wetherell $J$, Gatz M. Depression in older adults. Annu Rev Clin Psychol. 2009;5:363-89.

7. Alexopoulos GS. Mechanisms and treatment of late-life depression. Transl Psychiatry. 2019;9(1):1-16.

8. Huang AX, Delucchi K, Dunn LB, Nelson JC. A systematic review and metaanalysis of psychotherapy for late-life depression. Am J Geriatr Psychiatry. 2015;23(3):261-73 
9. McGovern AR, Kiosses DN, Raue PJ, Wilkins VM, Alexopoulos GS Psychotherapies for late-life depression. Psychiatr Ann. 2014;44(3):147-52.

10. DGPPN B, KBV A, AkdÄ B, BApK D, DEGAM D, DGPs D. [German Guideline Unipolar Depression] S3-Leitlinie/Nationale VersorgungsLeitlinie Unipolare Depression-Langfassung, 2. Auflage. Version 5. Retrieved from www. leitlinien.de/nvl/depression; 2015.

11. Raue PJ, McGovern AR, Kiosses DN, Sirey JA. Advances in psychotherapy for depressed older adults. Curr Psychiatry Rep. 2017;19(9):57.

12. Olfson M, Blanco C, Marcus SC. Treatment of adult depression in the United States. JAMA Intern Med. 2016;176(10):1482-91.

13. Kessler E-M, Blachetta C. Age cues in patients' descriptions influence treatment attitudes. Aging Ment Health. 2018:1-4.

14. Laidlaw K, Pachana NA. Aging, mental health, and demographic change: challenges for psychotherapists. Prof Psychol Res Pract. 2009;40(6):601.

15. Choi NG, Lee A, Goldstein M. Meals on wheels: exploring potential for and barriers to integrating depression intervention for homebound older adults. Home health care services quarterly. 2011;30(4):214-30.

16. Gutzmann H, Schäufele M, Kessler E-M, Rapp M. Psychiatrische und psychotherapeutische Versorgung von Pflegebedürftigen. In: Jacobs K Kuhlmey A, Greß S, Schwinger A, editors. Pflegereport 2017. Stuttgart: Schattauer; 2017. p. 107-18.

17. Luck-Sikorski C, Stein J, Heilmann K, Maier W, Kaduszkiewicz H, Scherer M, et al. Treatment preferences for depression in the elderly. Int Psychogeriatr. 2017;29(3):389-98

18. Raue PJ, Weinberger MI, Sirey JA, Meyers BS, Bruce ML. Preferences for depression treatment among elderly home health care patients. Psychiatr Serv. 2011;62(5):532-7.

19. Choi NG, Sirey JA, Bruce ML. Depression in homebound older adults: recent advances in screening and psychosocial interventions. Curr transl Geriatrics Experiment Gerontol Rep. 2013;2(1):16-23.

20. Carpenter B, Ruckdeschel K, Ruckdeschel H, Haitsma KV. REM psychotherapy: a manualized approach for long-term care residents with depression and dementia. Clin Gerontol. 2003;25(1-2):25-49.

21. Meeks S, Looney SW, Van Haitsma K, Teri L. BE-ACTIV: a staff-assisted behavioral intervention for depression in nursing homes. Gerontologist. 2008;48(1):105-14.

22. Gellis ZD, McGinty J, Horowitz A, Bruce ML, Misener E. Problem-solving therapy for late-life depression in home care: a randomized field trial. Am J Geriatr Psychiatry. 2007;15(11):968-78.

23. Laidlaw K, Kishita N. Age-appropriate augmented cognitive behavior therapy to enhance treatment outcome for late-life depression and anxiety disorders. GeroPsych. 2015;28(2):57-66.

24. Wang JJ. The effects of reminiscence on depressive symptoms and mood status of older institutionalized adults in Taiwan. Int J Geriatric Psychiatry. 2005;20(1):57-62.

25. Sutcliffe C, Cordingley L, Burns A, Mozley CG, Bagley H, Huxley P, Challis D. A new version of the geriatric depression scale for nursing and residential home populations: the geriatric depression scale (residential) (GDS-12R). International Psychogeriatrics. 2000;12(2):173-81.

26. Gauggel S, Birkner B. Validität und Reliabilität einer deutschen Version der Geriatrischen Depressionsskala (GDS). Zeitschrift für Klinische Psychologie und Psychotherapie. 1999;28(1):18-27

27. Kessler E-M. PSY-CARE manual - a guide for needs-based psychotherapeutic work with older adults living at home who need care for cognitive behavioral therapists. In: PSY-CARE manual - Ein Leitfaden für edarfsangemessenes psychotherapeutisches Arbeiten mit zuhauselebenden älteren menschen mit Pflegebedarf für kognitive Verhaltenstherapeut*innen. Berlin: MSB Medical School Berlin; 2019.

28. Kessler E-M. [PSY-CARE guide for older people with care needs and depression and their relatives] PSY-CARE Ratgeber für ältere Menschen mit Pflegebedarf und Depression und ihre Angehörigen. Berlin: MSB Medical School Berlin; 2019.

29. Bäcker G. Reform of the long-term care insurance in Germany. In: European Social Policy Network ESPN Flash Report 2016/43; 2016.

30. Hautzinger M. [help for those affected and their families with depression in old age] Wenn Ältere schwermütig werden: Hilfe für Betroffene und Angehörige bei Depression im Alter: Beltz; 2006.

31. Sutcliffe C, Cordingley L, Burns A, Mozley CG, Bagley H, Huxley P, Challis D. A new version of the geriatric depression scale for nursing and residential home populations: the geriatric depression scale (residential) (GDS-12R). Int Psychogeriatr. 2000;12(2):173-81.
32. Gauggel S, Birkner B. Validität und Reliabilität einer deutschen Version der Geriatrischen Depressionsskala (GDS). Z Klin Psychol Psychother. 1999;28(1):18-27.

33. Conrad I, Matschinger H, Kilian R, Riedel-Heller S. WHOQOL-OLD und WHOQOL-BREF - Handbuch für die deutschsprachigen Versionen der WHO-Instrumente zur Erfassung der Lebensqualität im Alter. Göttingen: Hogrefe; 2016.

34. Margraf J. Mini-DIPS: Diagnostisches Kurz-Interview bei psychischen Störungen (Mini-DIPS: short diagnostic interview for mental disorders) Berlin, Heidelberg, New York, Tokio: Springer; 1994.

35. Hinz A, Brähler E. Normative values for the hospital anxiety and depression scale (HADS) in the general German population. J Psychosom Res. 2011 71(2):74-8.

36. Mahoney Fl, Barthel DW. Functional evaluation: the Barthel index: a simple index of independence useful in scoring improvement in the rehabilitation of the chronically ill. Maryland State Med J. 1965;14:61-5.

37. Lawton MP, Brody EM. Assessment of older people: self-maintaining and instrumental activities of daily living. Gerontologist. 1969;9(3):179-86.

38. Faul F, Erdfelder E, Buchner A, Lang A-G. Statistical power analyses using $\mathrm{G}^{*}$ power 3.1: tests for correlation and regression analyses. Behav Res Methods. 2009;41(4):1149-60.

39. Cooper AA, Conklin LR. Dropout from individual psychotherapy for major depression: a meta-analysis of randomized clinical trials. Clin Psychol Rev. 2015:40:57-65.

40. Kessler E-M. PSY-CARE Manual (Version 1.0). unveröffentlichtes Schulungsmanual; 2019.

41. R Core Team. R Foundation for Statistical Computing; Vienna, Austria: 2014. R: A language and environment for statistical computing 2015.

42. R. R: A language and environment for statistical computing. R Foundation for Statistical Computing. Vienna: R Core Team; 2014.

\section{Publisher's Note}

Springer Nature remains neutral with regard to jurisdictional claims in published maps and institutional affiliations.
Ready to submit your research? Choose BMC and benefit from:

- fast, convenient online submission

- thorough peer review by experienced researchers in your field

- rapid publication on acceptance

- support for research data, including large and complex data types

- gold Open Access which fosters wider collaboration and increased citations

- maximum visibility for your research: over $100 \mathrm{M}$ website views per year

At $\mathrm{BMC}$, research is always in progress.

Learn more biomedcentral.com/submissions 